\title{
Les Libanais de Guyane : un modèle d'identifications multiples
}

Par Isabelle Dubost, anthropologue, MCF, université des Antilles et de la Guyane, CRPLC ${ }^{(1)}$

Originaires du même village dans les montagnes, Bazoun, les Libanais se sont installés dès la fin du XIXe siècle en Guyane. Des migrations continues et des échanges entretenus avec

la famille restée au pays maintiennent un fort sentiment d'appartenance à la culture d'origine. 
La Guyane, terre continentale, est l'interface entre Amérique latine et Caraïbe. Une histoire coloniale et esclavagiste la relie à ce vaste archipel, et plus encore à la Guadeloupe et à la Martinique, du fait d'une appartenance commune à l'entité appelée "ultramarine" ou "ultrapériphérique", cet ensemble des départements français d'Amérique.

Ce département qui se veut aussi "pays" est en pleine effervescence, dans une période de réajustement et de rééquilibrage tout à la fois économique, social et ethnique. C'est le temps d'un repositionnement des différents groupes dans l'ensemble guyanais, chacun tentant de trouver une place privilégiée et surtout une reconnaissance sociale, voire institutionnelle. En effet, l'équilibre traditionnel entre les différents groupes est bouleversé par les mouvements migratoires récents et par l'évolution démographique. Dès lors, la problématique fondamentale de la société guyanaise est devenue celle de la cohabitation de nombreuses populations aux intérêts divergents et parfois contradictoires. Quelles sont les conditions de ce possible ? Jusqu'à quel point est-il possible de concilier le divers et le particulier? Dans ce contexte, comment les Guyanais originaires du Liban se positionnent-ils? Comment ancrent-ils leur système d'identifications?

Originaires de Bazoun, dans les montagnes, et, pour quelques-uns, de Zghorta, des Libanais se sont installés dès la fin du $\operatorname{XIX}^{e}$ siècle en Guyane. Des migrations continues et des échanges entretenus avec la famille restée au pays maintiennent un sentiment d'appartenance à la culture d'origine. Pourtant, ces Libanais sont aussi des Guyanais et revendiquent leur place dans la société par une activité commerciale importante et un rôle majeur au sein des institutions professionnelles représentatives. C'est donc par l'action économique que les Libanais entendent se positionner et non par des revendications particularistes d'ordre ethnique. Leur univers est aussi celui de la mobilité dans différents espaces: le Liban, la France, la Caraïbe - plus précisément la Martinique et la Guadeloupe, le Suriname, qui constituent des points d'ancrage pour des échanges familiaux et commerciaux pouvant être encore plus diversifiés. Cette mobilité permanente devient une ressource pour alimenter un sentiment d'appartenance culturelle originelle.

\section{La Guyane : terre d'immigration}

Alors que le Bassin caraïbe et ses bordures sont, selon Simon", "l'un des tous premiers pôles mondiaux de l'émigration", la Guyane se situe dans une tout autre perspective puisqu'elle est un territoire d'immigration. Ainsi, elle offre une expérience à grande échelle de rencontre entre différentes populations. 
Le destin guyanais s'est confronté dès l'origine à "l'hésitation gouvernementale, jamais dépassée, entre faire de ce pays une colonie de peuplement ou en faire une colonie d'exploitation ${ }^{* 33}$. Les premières migrations mènent à l'installation de colons et l'importation d'une main-d'cuvre servile pour travailler sur les habitations ${ }^{(4)}$. Des bagnards sont également contraints de vivre en Guyane. À partir de 1855, l'exploitation aurifère dans l'intérieur des

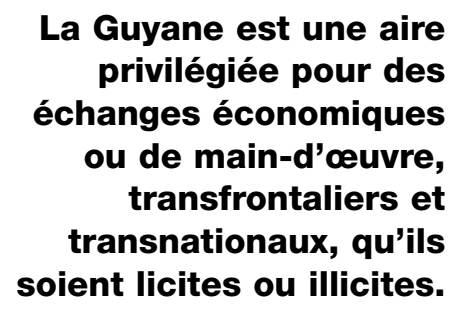

La Guyane est une aire privilégiée pour des échanges économiques ou de main-d'œuvre, transfrontaliers et transnationaux, qu'ils soient licites ou illicites. terres attire des populations originaires des Petites Antilles, principalement anglophones - Saintes-Luciens, Dominicains - et dans une moindre mesure francophones - Martiniquais et Guadeloupéens. En 1902, suite à l'éruption de la montagne Pelée, des Martiniquais trouvent refuge en Guyane. Dans la seconde moitié du XIX ${ }^{\mathrm{e}}$ siècle, ce sont des populations chinoises et libanaises qui viennent tenter leur chance. À partir des années cinquante, des plans de peuplement voient le jour afin de développer l'agriculture. Des Polonais, des Javanais vont alors se succéder sur le territoire. La Guyane a également accueilli des Hmongs originaires du Laos, dans les années soixante-dix.

Qu'en est-il aujourd'hui ? La population, forte de 191000 habitants, selon l'estimation de l'Insee, en 2005, se décline en 139 nationalités ${ }^{(5)}$. Bien que l'espace urbain concentre la plus grande partie de la population, les Autochtones, dits "peuples premiers", constitués de différents groupes d'Amérindiens et de Bushinenge ou Noirs Marrons ${ }^{(6)}$, vivent en forêt ou sur les fleuves. Le groupe des Créoles $^{(7)}$ est celui qui subit avec le plus d'intensité les bouleversements guyanais puisque "majoritaires jusque dans les années soixante-dix, ils ne représentent plus de nos jours qu'un tiers environ de l'ensemble de la population" ${ }^{48}$.

Les immigrés représentent $29 \%$ de la population ${ }^{(9)}$. Les Surinamiens constituent le premier groupe - 33,1 \% -, les Haïtiens le second - 28,2 \% - et les Brésiliens le troisième - 16,8 \%. Les Caribéens au sens large ont un impact relativement important puisqu'ils représentent "un tiers de la population totale et deux tiers des immigrés, constitués de Haïtiens pour les deux tiers, d'Antillais français, des ressortissants des deux autres Guyanes ainsi que de lîle de Sainte-Lucie ${ }^{\left.{ }_{110}\right)}$. Dans les années quatrevingt, les Surinamiens sont venus nombreux, à la suite de la guerre civile qui s'est terminée en 1992. Cette immigration est restée forte pour des raisons économiques et sociales ${ }^{(11)}$. L'immigration haitienne massive et clandestine, qui date des années soixante-dix, est en déclin depuis les années quatre-vingt. L'immigration brésilienne, qui débute dès 1965, est constituée de flux constants. 
De nombreux facteurs peuvent expliquer l'attirance qu'exerce la Guyane. Tout d'abord la proximité géographique avec le Suriname, le Brésil, le Guyana, et une proximité relative avec la Caraïbe. Entre la Caraïbe et la Guyane, il s'agit plus d'une proximité sociale et culturelle du fait d'un héritage historique commun liéà la colonisation, l'esclavage et l'économie de plantation, bien que ce dernier facteur n'ait jamais eu la même ampleur que dans la Caraïbe. Cette proximité est assortie d'une forte perméabilité des frontières, dans une zone essentiellement forestière donc difficilement contrôlable. Enfin, les conditions économiques favorables attisent la convoitise pour une vie meilleure. La Guyane est une aire privilégiée pour des échanges économiques ou de main-d'œuvre, transfrontaliers et transnationaux, qu'ils soient licites ou illicites. Les Libanais, quant à eux, ont investi le territoire guyanais dès la seconde moitié du XIX ${ }^{\mathrm{e}}$ siècle et y maintiennent leur mobilité.

\section{L’émigration libanaise}

L'immigration libanaise en Guyane ne peut être comprise hors de son contexte d'origine. L'histoire du Liban est très fortement liée à celle de la France. Après que la Haute Porte ottomane a accordé à la région du Mont Liban un régime particulier d'autonomie en 1864, le Règlement organique, administré par un gouverneur maronite $^{(12)}$, de 1920 à l'indépendance en 1943, le Liban et la Syrie forment alors le Grand Liban ou la Grande Syrie, sous mandat français, tandis que la Palestine est placée sous mandat britannique.

Les événements de 1860 - massacre des maronites par les Druzes ${ }^{(13)}$ - ont déclenché la première vague d'émigration : 100000 chrétiens en majorité maronites seraient partis. Une grave crise économique et démographique entraîne égalements d'autres départs : il s'agit notamment de la crise de la monoproduction de la soie et de la baisse des revenus des transits liés à la création du canal de Suez, assorties d'un accroissement rapide de la population ${ }^{(14)}$. Cette situation a donné lieu à une "composition de l'émigration libanaise de paysans et de petits propriétaires terriens" Parallèlement, l'attrait des pays en voie de développement, pourvoyeurs de travail pour des populations en difficulté, se confirme. Les "Amériques" deviennent alors une terre chimérique à atteindre.

La seconde vague d'émigration pendant l'entre-deux-guerres, qui touche des citadins plus instruits, s'oriente vers l'Amérique latine et l'Afrique, projet soutenu par la France, qui voyait les Libanais comme de bons intermédiaires commerciaux dans l'empire colonial ${ }^{(16)}$. Le bilinguisme de mise au Liban a vraisemblablement 
facilité ces migrations vers des pays francophones. De 1945 à 1975, la troisième vague change radicalement de composition: "Ce ne sont plus des pionniers qui s'en vont mais des gens qui s'en vont trouver à l'étranger des parents riches et influents qui prendront à leur charge le soin de leur trouver un travail. ${ }^{(17)}$ Une période de déclin s'ensuit, amorcée par des lois restrictives dans plusieurs pays, pour reprendre de l'ampleur durant la guerre civile entre 1975 et 1990.

L'émigration libanaise a beaucoup évolué: à l'origine religieuse et économique - les candidats au départ sont des paysans pauvres en quête d'une vie meilleure -, elle est restée religieuse mais est devenue aussi politique - ce sont maintenant les classes aisées et des intellectuels qui prennent le départ. Il existe pourtant une constante: ce sont essentiellement des maronites qui s'exilent. Toujours est-il que le taux d'émigration au Liban est l'un des plus élevés du monde : entre $30 \%$ et $40 \%$ en $1990^{(18)}$. On estime à 900000 environ le nombre de Libanais ayant quitté leur pays. Cette mobilité est dirigée encore aujourd'hui vers les États-Unis, le Brésil, l'Argentine, le Canada, l'Australie mais aussi vers l'Afrique occidentale et le Golfe.

\section{L'immigration libanaise en Guyane}

C'est dans ce contexte historique que se déroule l'immigration libanaise en Guyane. Forte, à la fin du XIX ${ }^{\mathrm{e}}$ siècle, elle se déroule de nos jours de manière continue mais faible. La migration de travail est devenue une immigration fondée sur le regroupement familial dans le but de consolider une entreprise et d'offrir ainsi à la famille de bonnes opportunités économiques.

D'après les entretiens, les familles se cotisent, vendent des terrains ou économisent durant des années afin que des hommes quittent leur terre natale pour la Guyane ou les Antilles, pour faire fortune et ensuite revenir. Tel est le projet migratoire. Une douzaine de pionniers arrivent vers 1895 puis entre 1905 et 1910. Pendant la seconde période, entre 1920 et 1940 puis dans les années cinquante, respectivement trois puis cinq pionniers s'installent. Ensuite, il s'agira d'immigration individuelle et ponctuelle, en 1966 et en 1976. Cette observation correspond d'ailleurs aux trois périodes d'émigration du Liban.

Les migrants sont tous des chrétiens maronites fuyant la misère car leur métier d'agriculteur - culture de l'olive dans les montagnes, fabrication d'huile et de savon - ne peut subvenir à leurs besoins. À partir de la guerre de 1975, des musulmans s'installent. La configuration de ces migrations montre une dispersion 
des familles en Amérique latine - Suriname, Brésil, Argentine, Venezuela, Colombie, Bolivie -, en Amérique centrale - Mexique... -, dans la Caraïbe - Trinidad, Dominique, Martinique, Guadeloupe, Antigua, Cuba, Porto Rico - et aux États-Unis.

Les parcours se déploient parfois en différents sites d'accueil, au gré des opportunités, avec des retours momentanés au Liban, le temps d'un mariage, d'une naissance ou lors de la guerre. Le voyage dure deux mois et demi et les escales varient selon les bateaux : les navires italiens transitent à Gênes et à Naples, les français à Marseille et les anglais à Liverpool, ce qui est parfois déterminant pour la destination finale. Parfois, ce sont des recruteurs commissionnés qui cherchent des candidats à l'exil.

La nationalité des migrants originaires d'un territoire ottoman était turque. Entre 1923 et 1926, le traité de Lausanne octroyait à ces migrants la possibilité d'opter pour la nationalité de leur pays d'origine. Cependant, le Liban étant alors sous mandat français, les ressortissants bénéficiaient du même régime

Les parcours se déploient
parfois en différents sites
d'accueil, au gré des
opportunités, avec des
retours momentanés au
Liban, le temps d'un
mariage, d'une naissance
ou lors de la guerre.
de protection que les Français. En 1931, les services des Colonies dénombrent 31 résidents libanais en Martinique, dont 26 hommes et 5 femmes, 180 en Guadeloupe dont 67 hommes, 50 femmes et 63 enfants : tous y gardent leur nationalité ; en Guyane, par contre, si 124 résidents sont dénombrés, dont 63 hommes et 61 femmes, 84 Libanais optent pour la nationalité française. Ce sont donc au total 356 adultes qui sont recensés sur les colonies d'Amérique ${ }^{(19)}$.

Ces pionniers, âgés d'une trentaine d'années, sont souvent des frères. Soit ils viennent avec leurs épouses, soit ils partent seuls et font venir leurs femmes dès que les conditions économiques sont favorables à leur installation, ce qui correspond souvent à l'acquisition d'un commerce. Ils travaillent auprès d'un oncle propriétaire d'une boutique, auprès duquel ils font leur apprentissage. En 2004, seuls 22 ressortissants libanais sont recensés en Guyane ${ }^{(20)}$, dont deux enfants. Ce chiffre montre à quel point les Libanais présents sont devenus des Français, soit par naissance, soit par naturalisation.

Dans un pays comme celui-ci, fondé sur un contexte migratoire sans pareil, comment vivre ensemble ? Quelles stratégies adopter pour maintenir un sentiment d'appartenance à un groupe et une culture d'origine ? Ces stratégies relèvent-elles de préférences ethniques? 


\section{De l'ethnicité...}

Le concept d'ethnicité renvoie obligatoirement à celui de frontière et donc à la figure de l'étranger.

L'étranger vient de l'ailleurs, mais c'est aussi celui qui pose de la distance entre soi et les autres par son étrangeté, transparaissant de prime abord, et qui pose aussi de la ressemblance par son humanité, comme le dit Georg Simmel ${ }^{211}$. Il est ainsi le support d'une ambivalence: "Les modèles qui formalisent le rapport entre l'étranger et l'autochtone tiennent compte du conflit, de la juste résistance au changement et de la juste acceptation $d u$ changement qu'implique l'établissement $d u$ rapport. D'où l'ambivalence."(22) Il est à la fois "proche et lointain, mobile et stable, marginalisé pour des raisons affectives, intégré pour des raisons d'intérêt." ${ }^{\text {233) }} \mathrm{Il}$ crée aussi le "besoin simultané de réaffirmer la fidélité à l'identité collective et de se pencher hors de son enceinte protectrice pour saisir ce que le monde de l'appartenance ne saurait offrir." ${ }^{\text {(24) }}$

C'est ce mode ambivalent de l'altérité qui va permettre de comprendre comment un groupe s'identifie et comment les autres l'identifient. Selon Rogers Brubaker, en effet : "L'auto-identification et l'identification de l'autre sont fondamentalement des actes situationnels et contextuels. ${ }^{25)}$

Au-delà d'un contexte déterminant, ces modalités d'identification relèvent de l'ethnicité, qui pourrait se définir, selon Pierre-Jean Simon, comme "un ensemble de traits relativement objectifs - ou du moins objectivables - partagés par une pluralité d'individus et les constituant en une collectivité particulière, relativement cohérente, et, en même temps, une conscience commune, un sentiment partagé par un ensemble d'acteurs sociaux d'appartenance à cette collectivité, et leur identification - plus ou moins - à cette collectivite' ${ }^{\prime 26}$. Cela permet de comprendre les processus d'inclusion et d'exclusion, la frontière donnant lieu à une catégorisation des groupes selon des critères variables, comme l'origine, le statut majoritaire ou minoritaire... Or cette frontière renvoie à l'unité : "Ce sont des principes de division qui lorsqu'ils simposent à l'ensemble d'un groupe font le sens et le consensus sur le sens, et en particulier sur lidentité et l'unité du groupe. ${ }^{\text {,27) }}$ Mais la frontière qui rapproche est aussi celle qui maintient de la distance. Ainsi : "analyser l'ethnicité c'est rendre compte de l'ensemble des pratiques de différenciation qui instaurent et maintiennent une frontière ethnique", au sens de Barth, "et non restituer le substrat culturel couramment associé à un groupe ethnique en tant que contenu de nature éternelle et stable... Il convient alors de reconnaître que n'existe pas d'identité hors de l'usage qui en est fait, ni de substrat culturel invariant qui définirait, hors de l'action sociale, l'essence d'un membre d'un groupement humain particulier ${ }^{2128}$.

Ces pratiques de différenciation sont induites par les acteurs, selon Barth ${ }^{(29)}$ : "Les groupes ethniques sont des catégories d'attribution et d'identification opérées par les 
acteurs eux-mêmes et ont donc la caractéristique d'organiser les interactions entre les individus." Nous sommes dans le mode de la représentation puisque "les groupes n'existent que dans la mesure où ils sont faits, et sans cesse refaits, par les affirmations de tous ceux qui en proclament l'existence. Ces affirmations sont l'expression des stratégies d'identification par lesquelles les individus, en interaction avec d'autres et selon un contexte social donné, se coalisent et se distinguent, se regroupent et s'opposent, bref se constituent - dans et par l'action - en groupes, en exposant une appartenance ethnique contraire à d'autres appartenances du même type ?’30)

Quelles sont alors les catégories qui vont rassembler et séparer les Libanais des autres Guyanais ? Où se situent les frontières avec les autres groupes?

Il s'avère que les Libanais mobilisent des ressources ethniques, mais n'ont aucune stratégie d'ethnicisation pour se démarquer de manière radicale des autres, contrairement aux Créoles, comme l'ont montré les travaux de Jolivet et d'Hidair ${ }^{(31)}$. Groupe devenu minoritaire et pourtant détenteur d'un pouvoir politique fort, alors que l'émergence politique des Amérindiens et des Noirs Marrons est de plus en plus marquée, la difficulté pour les Créoles est de maintenir leur position en toute légitimité( ${ }^{-(2)}$.

Le choix des Libanais est tout autre. Ils fondent leur système d'appartenance sur des réseaux. C'est donc un groupe ouvert au monde, acteur de ce "Tout-Monde" cher à Glissant, un groupe en mobilité constante tout en étant là. Sa référence territoriale à laquelle s'identifier, au-delà de la Guyane, est celle d'un réseau mondialisé, le maillage familial demeurant la pierre angulaire de ce système d'appartenance sociale.

\section{L'entrepreneuriat}

Les Libanais, dès leur arrivée en Guyane, se sont spécialisés dans le commerce de proximité, en vendant des produits domestiques (linge, tissu, mercerie, casseroles, vitrerie, literie...). Quelques-uns ont été colporteurs avec des charrettes - à bras ou mues par des animaux -, durant une courte période de quatre à cinq ans, mais cela n'est pas aussi significatif qu'aux Antilles. Fin xix ${ }^{\mathrm{e}}$ siècle, leurs boutiques sont dispersées le long des habitations et le long des routes à Rémire-Montjoly, Macouria, Kourou. Ils saisissent l'opportunité de la ruée vers l'or en installant des magasins dans les zones aurifères, comme à Mana, proposant des articles de quincaillerie pour les chercheurs d'or. Petit à petit, une diversification est amorcée avec la confection et le prêt-à-porter, la lingerie, la parfumerie. Aujourd'hui, des établissements tentent de maintenir une activité rentable, en concurrence avec les 
magasins franchisés de confection ou de sport. Des Libanais sont également concessionnaires de grandes marques automobiles comme Mercedes, Mazda... La restauration demeure une activité peu développée. Ceux qui quittent l'univers commercial s'orientent vers des professions libérales, devenant médecins, dentistes, avocats, ou embrassent des carrières dans la fonction publique, tandis que les plus fortunés investissent dans l'immobilier.

L'organisation de ces entreprises est strictement familiale. Ce sont des frères, des époux, des parents et leurs descendants qui s'associent, bien souvent à parts égales. Par exemple, lorsqu'un groupe développe plusieurs magasins, chaque membre de la famille devient gérant d'une structure. Si celle-ci nécessite des cadres, des frères ou des cousins restés au pays migrent afin de pallier ce déficit en main-d'ceuvre. Cette nécessité entraîne un flux restreint mais continu de migrants. L'équipe directionnelle est donc familiale alors que les employés sont d'appartenances culturelles diverses : créole, brésilienne, surinamienne, métropolitaine... Cette présence familiale est indispensable car, d'après un propriétaire de quinze magasins et d'une société de gestion, "on travaille beaucoup, donc seule la famille peut s'engager". Arrivé seul en 1986 pour travailler chez un oncle en Guadeloupe puis en Guyane, ce migrant a fait venir à son tour ses frères et sceurs, ses cousins... Parfois, les enfants ne sont pas rémunérés, ce qui est aussi observable dans les familles chinoises. Associés, ils prennent de l'argent dans la caisse selon leurs besoins. Les sommes amassées peuvent alors constituer un capital pour une création d'entreprise.

\section{Des frontières}

Aux yeux des Guyanais, les Libanais forment un groupe à part, sauf en période de carnaval, temps festif durant lequel les frontières disparaissent car les Libanais vont danser chez Paulina ${ }^{(33)}$. Il s'avère pourtant que des frontières fortes existent au sein de ce groupe, ce qui induit des catégories sociales et religieuses bien distinctes. Les relations sociales sont limitées au réseau familial élargi. Certes, l'interconnaissance est de rigueur, chacun est identifié selon son appartenance, ses alliances familiales et son statut social et économique qui va de pair, mais il est de mise de se tenir éloigné de ses compatriotes, qui plus est lorsqu'ils ne sont pas de même confession. Ainsi, les maronites ne côtoient pas les musulmans.

Aucune association ne vient structurer le groupe, malgré une tentative. Aucune activité particulière ne permet de rencontres. De religion maronite dans leur majorité, les Libanais fréquentent les églises catholiques. Seule la statue de saint Charbel, originaire du nord du Liban près de Bazoun, déposée à l'église Saint- 
Antoine à Zéphir, est vénérée par des pratiquants et les rassemble lors d'un anniversaire, le 24 décembre.

Les Libanais ne se fréquentent pas, sauf lors de grandes manifestations : deuil, mariage, naissance... Sinon, on se reçoit en famille, le dimanche, pour déjeuner. En fait, on privilégie une attitude de distanciation. Il est très souvent fait référence, lors des entretiens, à des relations de jalousie et de mesquinerie, voire de rivalité exacerbée, qui mettent en scène une concurrence professionnelle. Tous les Libanais n'ont pas la même réussite sociale. Fréquents sont les énoncés comme: "le Libanais est l'ennemi du Libanais, il n'y a pas de communauté ici, pas d'entraide." La famille devient alors une sorte de refuge.

Les Libanais qui ont franchi de manière radicale la frontière les séparant des Créoles sont exclus : on condamne ceux qui épousent des non-Libanais et leurs enfants métis subissent la même sanction, car le mariage préférentiel demeure ethnique - on condamne également ceux qui ne se conforment pas à la culture libanaise : la première femme à avoir lancé une procédure de divorce a été totalement bannie. Ainsi, l'unité du groupe est confortée, dès lors qu'une sanction s'applique.

\section{Des réseaux}

Les réseaux commerciaux ne sont pas tissés sur une appartenance exclusivement libanaise. C'est l'intérêt économique qui prime dans les relations avec les fournisseurs et les fabricants. Ces réseaux se déploient dans un espace mondialisé entre l'Asie - Singapour, Chine, Hong Kong, Inde -, l'Amérique latine - Colombie, Suriname, Brésil -, la Caraïbe - Trinidad, Guadeloupe, Saint -Domingue, la Barbade - et l'Europe - plus particulièrement, la France, avec Paris, Lyon et Marseille. Des intermédiaires sont commissionnés, mais bien souvent ce sont les propriétaires qui font le déplacement plusieurs fois dans l'année, poursuivant leur périple jusqu'au Liban, ce qui leur permet de rencontrer des membres de leur famille. Il n'existe aucune forme de tontine d'ordre ethnique afin de financer une création d'entreprise, seule la famille a cette charge.

Des échanges familiaux sont fréquents à une large échelle territoriale. La famille étant dispersée, on se visite lors des vacances, on part en week-end au Suriname, si proche, où une communauté libanaise est bien structurée. La mobilité est donc constante. Chaque famille part au minimum une fois par an au Liban.

Le village est au centre d'un maillage territorial. Il est le lieu fondateur de la lignée familiale, la référence culturelle imaginée et permet de réactiver celle-ci. Bazoun est une commune de montagne où le climat rude oblige la population à migrer 
pendant une partie de l'année à Dahrer-El-Ain. Chaque famille a conservé une maison et des biens dans chacun des villages. Un membre de la famille ou une personne mandatée est chargé de la gestion et de l'exploitation des terres agricoles. Aucun revenu n'est d'ailleurs versé aux propriétaires. Il ne s'agit pas de fermage, mais d'entretien. Au fur et à mesure de la réussite sociale dans le cadre de la migration, la maison familiale sera rénovée, une nouvelle construite, ce qui entraîne parfois des problèmes d'héritage liés à l'indivision.

Des liens forts sont donc maintenus avec le "pays". Deux mille personnes ${ }^{(34)}$ vivent encore sur place tandis que deux mille vivent à l'étranger, les enfants ne sont pas toujours répertoriés. Par contre les mariages sont souvent légalisés au Liban. Proche dans les cceurs, le village est donc tout aussi proche par des échanges réguliers.

\section{L'expérience libanaise : des identifications multiples}

Les Libanais partagent un système d'appartenances multiples. L'appartenance guyanaise est la référence importante, tout comme la nationalité française, pour certains, tandis que la référence libanaise est fondatrice : elle est d'ordre culturel. Lorsque des élections ont lieu, une mobilisation, certes ponctuelle, émerge pour un candidat d'origine libanaise. Le sentiment partagé est alors celui de la "fierté" de voir l'un des leurs accéder à une position de pouvoir. Cette importance de l'origine est notable car ceux qui sont au ban du groupe ressentent la vive souffrance de l'exclusion. Un Libanais âgé d'une quarantaine d'années, naturalisé depuis 1984, affirmait : "Je suis libanais, le Liban est mon pays, la France est ma patrie et je suis $100 \%$ guyanais!"

Ce sentiment d'appartenance libanaise est réactivé par la langue : le libanais est de rigueur à la maison et entre compatriotes. La transmission de la langue aux jeunes générations est plus difficile aujourd'hui et n'est pas liée à l'ancienneté sur le territoire guyanais. Chaque famille fait son choix. Le créole est la langue du commerce et des liens de socialisation à l'école ; le français est également parlé en famille. Un signe d'appartenance fort est décelable au cimetière puisque des inscriptions sur les tombes indiquent le lieu de naissance au Liban.

Il n'en demeure pas moins que l'implication d'ordre économique et social dans l'espace guyanais est fondamentale. Des acteurs politiques jouent des rôles importants au sein des conseils général et régional, dans les conseils municipaux de Cayenne, de Sinnamary, de Saint-Laurent, d'autres sont également présents 
dans les instances professionnelles - comme l'Ugic, le syndicat des commerçants, ou la chambre de commerce.

L'expérience libanaise en Guyane nous montre qu'il est possible de se positionner sur plusieurs registres d'appartenance, fonctionnant à des niveaux différents, ayant des fonctions différentes, mais tous jouant un rôle structurant.

Dans un univers guyanais travaillé par ces problématiques ethniques et de revendications particularistes, "il y a matière à s'interroger sur la viabilité du mouvement d'ethnicisation amorcé : expression d'une crise identitaire comme en connaissent beaucoup de peuples confrontés au progrès à l'occidentale, il n'est peut-être qu'un moment dans un processus de plus longue haleine, où la dynamique créatrice, celle dont sont précisément nées les cultures créoles, pourrait retrouver toutes ses prérogatives. Resterait toutefois à résoudre l'intégration des Guyanais relevant de sociétés plus holistes: est-elle possible en d'autres termes que ceux d'une créolisation ?’35)

Les Libanais guyanais n'ont pas de revendication particulariste, si ce n'est des exigences professionnelles afin de défendre le statut des commerçants. Il n'y a rien d'ethnique dans cette mobilisation politique au sein des institutions représentatives du commerce. Leur volonté est de poursuivre une adaptation en Guyane et de s'y investir tout en maintenant une position sociale et économique de réussite. Chose peu aisée, du fait des difficultés économiques rencontrées avec le déploiement de grandes surfaces qui rivalisent durement avec le commerce de proximité et la venue de Chinois du Zhejiang, ressentie souvent comme une concurrence déloyale. Leur position identitaire est celle de la discrétion, même si leur intégration à la société guyanaise en fait des acteurs à part entière. Leur expérience est digne d'intérêt car elle offre une posture ethnique particulière, maintenant une frontière modulable selon les situations, la catégorie communautaire se fondant essentiellement sur le mariage. Cette posture introduit ainsi une réflexion sur le possible d'une pluriethnicité guyanaise, pour ainsi constituer la "communauté imaginée" guyanaise dont parle Gérard Collomb ${ }^{(36)}$.

\section{Notes}

1. Centre de recherche sur les pouvoirs locaux dans la Caraibe, université des Antilles et de la Guyane, Martinique.

2. Simon, Gildas, 1995 , p. 61.

3. Jolivet, 1990, pp. 11-32.

4. La mise en valeur de cette colonie, dans un premier temps l'île de Cayenne, fut limitée puisqu'elle ne produisait au

$\mathrm{XVIII}^{\mathrm{e}}$ siècle que quelques denrées : café, rocou, cacao, coton, sucre et, plus tard, bois.

5. Atlas des populations immigrées en Guyane, Insee-Acse, 2006.

6. Descendants des esclaves rebelles ayant fui les grandes plantations du Suriname aux XVII ${ }^{\mathrm{e}}$ et XVIII ${ }^{\mathrm{e}}$ siècles.

7. "Le principe constitutif (pour être désigné créole) est d'avoir l'esclavage comme situation fondatrice" et d' "être né sur place, d'une lignée originaire d'ailleurs [comme] une condition nécessaire mais non suffisante", même s'il demeure une grande ambiguïté dans la définition de ce terme, voir Jolivet, 1990. 


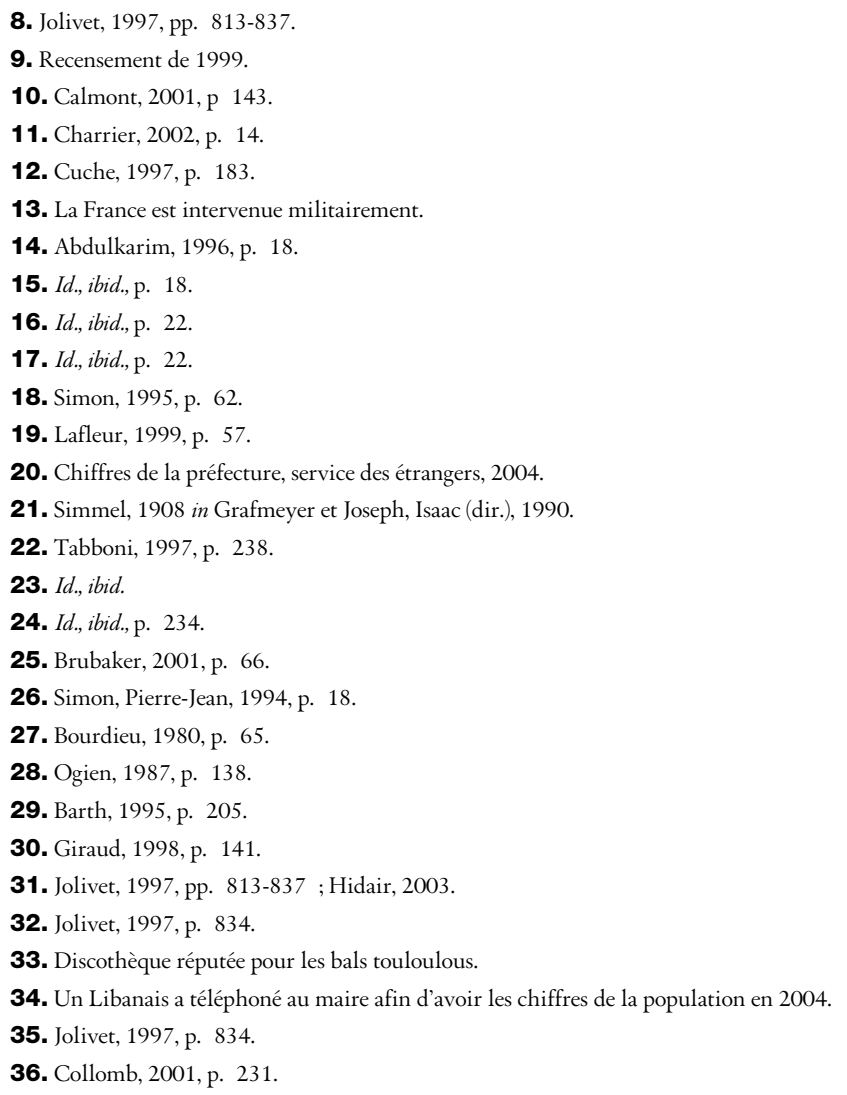

\section{Références bibliographiques}

- Abdulkarim, Amir, La Diaspora libanaise en France. Processus migratoire et économie, L'Harmattan, Paris, 1996.

Insee-Acse, Atlas des populations immigrées en Guyane, 2006.

- Barth, Fredrik, "Les groupes ethniques et leurs frontières", in Théories de l'ethnicité, Poutignat, Philippe et Streiff-Fenart, Jocelyne (dir.), PUF, Paris, 1995.

- Bourdieu, Pierre, "L'identité et la représentation. Éléments pour une réflexion critique sur l'idée de région", Actes de la recherche en sciences sociales, $\mathrm{n}^{\circ} 35$, nov. 1980, pp. 63-72.

- Brubaker, Rogers, "Au-delà de l'identité", Actes de la recherche en sciences sociales, n 139, sept. 2001, pp. 66-85.

- Calmont, André, "Flux et polarisations dans le Bassin caribéen", Relire l'histoire et la géographie de l'espace caribéen, Abenon,

L. ; Bégot, D. ; Bégot, M. ; Burac, M. ; Calmont, A. ; Hartog, T. (dir.), Hachette, Paris, 2001.

- Charrier, Rémy, "Guyane, des peuples et des histoires", AntianeÉco, n 54, 2002, pp. 14-17.

- Collomb, Gérard, "'Chassez le métissage..., notes à partir de la Guyane”, les Paradoxes du métissage, in actes du $123^{\mathrm{e}}$ congrès national des sociétés historiques et scientifiques, Antilles-Guyane (dir. Pierre Guillaume), 1998, éd. du CTHS, Paris, 2001, pp. 225-232.

- Cuche, Denys, "L'Immigration libanaise au Pérou : une immigration ignorée", Journal de la Société des américanistes, $\mathrm{n}^{\circ} 83$, 1997, pp. 173-199.

- Giraud, Michel, "L'ethnicité comme nécessité et comme obstacle", in Ferréol, Gilles (dir.), Intégration, lien social et citoyenneté, Presses universitaires du Septentrion, Lille, 1998. 
- Hidair, Isabelle, La Construction identitaire des Créoles de Guyane. De la gestion d'un héritage pluriel à l'insertion dans un contexte multiculturel, thèse d'anthropologie, Jolivet, Marie-José (dir), EHESS, 2003.

- Jolivet, Marie-José, "Entre autochtones et immigrants : diversité et logiques des positions créoles guyanaises", Études créoles, vol. XIII, n 2, 1990, pp. 11-32.

- Jolivet, Marie-José, "La créolisation en Guyane", Cahiers d'études africaines, vol. Xxxvil-4, n 148, 1997, pp. 813-837.

- Jolivet, Marie-José, "Mémoires guyanaises : fluctuations des représentations créoles du passé", Identités caraïbes, in actes du $123^{\mathrm{e}}$ congrès national des sociétés historiques et scientifiques, Antilles-Guyane (dir. Pierre Guillaume), 1998, éd. du CTHS, Paris, 2001, pp. 63-73.

- Lafleur, Gérard, Les Libanais et les Syriens de Guadeloupe, Paris, Karthala, 1999.

- Ogien, Albert, "Les usages de l'identité", in Vers des sociétés pluriculturelles :études comparatives et situations en France, actes de l'Association française des anthropologues (AFA), Paris, éd. de l'Orstom, 1987, pp. 133-139.

- Simmel, Georg, "Digressions sur l'étranger", 1908, in L'École de Chicago, naissance de l'écologie urbaine, Grafmeyer, Yves, Joseph, Isaac (dir.), Aubier, Paris, 1990 ( $2^{\mathrm{e}}$ éd.), 1984 (1 ${ }^{\text {re }}$ éd.).

- Simon, Gildas, Géodynamique des migrations internationales dans le monde, PUF, Paris, 1995.

- Simon, Pierre-Jean, "Ethnicité", Pluriel-recherches, cahier n², L'Harmattan, Paris, 1994.

- Tabboni, Simonetta, "Le multiculturalisme et l'ambivalence de l'étranger", in Une société fragmentée ? Le multiculturalisme en débat, Wieviorka, Michel (dir.), La Découverte, Paris, 1997. 